\title{
A NOTE ON INVARIANT FINITELY ADDITIVE MEASURES
}

\author{
S. G. DANI ${ }^{1}$
}

\begin{abstract}
We show that under certain general conditions any finitely additive measure which is defined for all subsets of a set $X$ and is invariant under the action of a group $G$ acting on $X$ is concentrated on a $G$-invariant subset $Y$ on which the $G$-action factors to that of an amenable group. The result is then applied to prove a conjecture of $\mathrm{S}$. Wagon about finitely additive measures on spheres.
\end{abstract}

It is well known that if $G$ is an amenable group acting on a set $X$ then there exist plenty of $G$-invariant finitely additive probability measures on $(X, \mathfrak{P}(X))$ where $\mathfrak{P}(X)$ is the class of all subsets of $X$ (cf. $[\mathbf{3}]$ for details). However, such measures may fail to exist when $G$ is nonamenable. In $[6] \mathrm{S}$. Wagon conjectured that if $G$ is a group of isometries of $S^{n}$, the $n$-dimensional sphere, such that for any $G$ invariant subset $Y$, the group $\{g / Y \mid g \in G\}$ of restrictions of elements of $g$ to $Y$ is nonamenable, then there does not exist any $G$-invariant finitely additive probability measure on $\left(S^{n}, \mathfrak{P}\left(S^{n}\right)\right)$ :

In this note we establish the above-mentioned conjecture. Further, we formulate a condition on actions of (abstract) groups, involving the isotropy subgroups and fixed point sets, which implies similar assertions in a more general situation (cf. Theorem 1.1). The condition holds for actions of subgroups $G$ of any compact Lie group $\mathfrak{G}$ acting on homogeneous spaces of $\mathfrak{G}$. It also holds for actions of subgroups $G$ of algebraic $\mathbf{R}$-groups $\mathfrak{G}$ acting on homogeneous spaces of $\mathfrak{G}$ by algebraic $\mathbf{R}$ subgroups. Thus, in all these cases we are able to conclude that $G$-invariant finitely additive probability measures (defined for all subsets) are concentrated on invariant sets on which the action factors to that of an amenable quotient of $G$ (cf. $\S 2$ ).

A particular consequence is that if $G$ is a nonamenable subgroup of $\operatorname{GL}(n+1, \mathbf{R})$ acting irreducibly on $\mathbf{R}^{n+1}$, then for the natural $G$-actions on $\mathbf{R}^{n+1}-(0), S^{n}$ or $\mathbf{P}^{n}$, there exist no invariant finitely additive probability measures (cf. Corollaries 2.2 and 2.4). We recall that by a theorem of A. Tarski $[5]$ this is equivalent to existence of paradoxical decompositions for the action (cf. [6 and $\mathbf{7}]$ for motivation and some results in that direction).

In some of the $G$-actions discussed above, e.g. $G$ a group of isometries of $S^{n}$ or $G$ a subgroup of a compact Lie group $\mathfrak{G}$ acting on a homogeneous space of $\mathfrak{G}$, there exists a natural countably additive $G$-invariant probability measure defined on the class of Borel subsets. We prove that the measure extends to a $G$-invariant finitely additive measure defined on all subsets if and only if $G$ is amenable (cf. Theorem

Received by the editors January 10, 1984.

1980 Mathematics Subject Classification. Primary 28A70.

Key words and phrases. Invariant finitely additive measures, amenable groups.

${ }^{1}$ Supported in part by NSF MCS-8108814 (A02). 
1.4 for a general result). For the case of groups of isometries of $S^{n}$ this was first proved by S. Wagon [6].

In $[\mathbf{1}]$ the author obtained results analogous to those discussed above for the case of subgroups of $\mathrm{GL}(n, \mathbf{Z})$ acting on $\mathbf{T}^{n}$ as group automorphisms. The present method is analogous but much simpler. In the case of the action on $\mathbf{T}^{n}$ the condition of Theorem 1.1 is not satisfied and consequently the proofs depend on various specific features of $\mathbf{T}^{n}$.

1. Main results. Let $G$ be a group acting on a set $X$. For $x \in X$ we denote by $G_{x}$ the isotropy subgroup under the $G$-action; viz. $G_{x}=\{g \in G \mid g x=x\}$. For any subgroup $H$ of $G$ we denote by $F_{H}$ the set of fixed points of $H$; that is $F_{H}=\{x \in X \mid h x=x$ for all $h \in H\}$.

For any set $E$ we denote by $\mathfrak{P}(E)$ the class of all subsets of $E$. A (possibly empty) subclass $\mathfrak{C}$ of $\mathfrak{P}(E)$ is said to be of type $\mathfrak{L}$ if the following holds: for any family $\left\{E_{\alpha}\right\}_{\alpha \in \Lambda}$, where $\Lambda$ is an indexing set and $E_{\alpha} \in \mathfrak{C}$ for all $\alpha \in \Lambda$, there exist $k \geq 1$ and $\alpha_{1}, \alpha_{2}, \ldots, \alpha_{k} \in \Lambda$ such that $\bigcap_{\alpha \in \Lambda} E_{\alpha}=\bigcap_{i=1}^{k} E_{\alpha_{i}}$.

ThEOREM 1.1. Let $G$ be a group acting on a set $X$. Suppose $\left\{G_{x} \mid x \in X\right\}$ and $\left\{F_{H} \mid H\right.$ a subgroup of $\left.G\right\}$ are of type $\mathfrak{L}$. Let $\mu$ be a $G$-invariant finitely additive probability measure on $(X, \mathfrak{P}(X))$. Then there exists a normal subgroup $Q$ of $G$ such that $G / Q$ is amenable and $\mu\left(F_{Q}\right)=1$.

We need the following lemma from [4] (cf. Proposition 3.5 of $[\mathbf{4}]$ ).

Lemma 1.2. Let $G$ be a group acting on a set $X$. Suppose that for all $x \in X$ the isotropy subgroup $G_{x}$ is amenable. Suppose also that there exists a $G$-invariant finitely additive probability measure $\mu$ on $(X, \mathfrak{P}(X))$. Then $G$ is amenable.

We first prove the following.

Proposition 1.3. Let $G$ be a group acting on a set $X$. Suppose $\left\{G_{x} \mid x \in X\right\}$ is of type $\mathfrak{L}$. Suppose also that there exists a $G$-invariant finitely additive probability measure $\mu$ such that for any nontrivial subgroup $H$ of $G, \mu\left(F_{H}\right)<1$. Then $G$ is amenable.

ProOf. If possible let $G$ be nonamenable. Let $\mathcal{N}$ be the class of all nonamenable subgroups $S$ of $G$ such that either $S=G$ or $S=\bigcap_{i=1}^{k} G_{x_{i}}$, where $k \geq 1$ and $x_{1}, \ldots, x_{k} \in X$. Let $\mathcal{M}$ be the subset of $\mathcal{N}$ consisting of minimal elements of $\mathcal{N}$; that is $M \in \mathcal{M}, N \in \mathcal{N}$, and $N \subset M$ implies $N=M$. Since the class of subgroups $\left\{G_{x} \mid x \in X\right\}$ is of type $\mathfrak{L}$, it follows that $\mathcal{M}$ is nonempty and that in fact any $N \in \mathcal{N}$ contains a minimal element (otherwise there would exist a sequence $x_{1}, \ldots, x_{k}, \ldots$, in $X$ such that $\bigcap_{i=1}^{n} G_{x_{i}}$ is a strictly decreasing sequence).

Let $M \in \mathcal{M}$. Being nonamenable, in particular $M$ is nontrivial. Hence, by hypothesis, $\mu\left(F_{M}\right)<1$ or, equivalently, $\mu\left(X-F_{M}\right)>0$. In particular, $X-F_{M}$ is nonempty. Consider the $M$-action on $X-F_{M}$. The isotropy subgroup of any $x \in X-F_{M}$ under the $M$-action is $M \cap G_{x}$ and it is a proper subgroup of $M$. We note that $M \cap G_{x}$ is amenable for all $x \in X$; if not, $M \cap G_{x} \in \mathcal{N}$, which would mean $M$ is not minimal in $\mathcal{N}$. On the other hand, on $X-F_{M}$ we have an $M$-invariant finitely additive probability measure $\nu$ defined by $\nu(E)=\mu(E) / \mu\left(X-F_{M}\right)$ for all $E \subset X-F_{M}$. By Lemma 1.2 these observations imply that $M$ is amenable-a contradiction. Hence $G$ must be amenable. 
ProOF OF THE THEOREM. Consider the class of subgroups $\mathfrak{C}=\left\{H \mid \mu\left(F_{H}\right)=\right.$ 1\}. Let $F=\bigcap\left\{F_{H} \mid H \in \mathfrak{C}\right\}$. Since $\left\{F_{H} \mid H \subset G\right\}$ is of type $\mathfrak{L}$ there exist $k \geq 1$ subgroups $H_{1}, \ldots, H_{k} \in \mathfrak{C}$ such that $F=\bigcap_{i=1}^{k} F_{H_{i}}$. Then $\mu(F)=1-\mu(X-F) \geq$ $1-\sum \mu\left(X-F_{H_{i}}\right)=1$. Let $Q$ be the subgroup generated by $\bigcup\{H \mid H \in \mathfrak{C}\}$. Since $\mu$ is $G$-invariant, $\mathfrak{C}$ is invariant under conjugation and consequently $Q$ is a normal subgroup of $G$. Further, clearly $F_{Q}=F$ so that $\mu\left(F_{Q}\right)=1$ and hence $Q$ is the unique maximal element of $\mathfrak{C}$. To complete the proof, we need only show that $G / Q$ is amenable. $F_{Q}$ is a $G$-invariant set and the action of $G$ on $F_{Q}$ factors to an action of $G / Q$. The restriction of $\mu$ to $F_{Q}$ is a $G / Q$-invariant finitely additive probability measure on $F_{Q}$. It is evident that the $G / Q$-action on $F_{Q}$ satisfies the conditions of Proposition $1.3\left(G / Q\right.$ and $F_{Q}$ in the place of $G$ and $X$ respectively). While the isotropy subgroups form a class of type $\mathfrak{L}$ because of the corresponding property for the $G$-action, the set of fixed points of any nontrivial subgroup has $\mu$-measure $<1$ because of our choice of $Q$ as the unique maximal element of $\mathfrak{C}$ as defined above. Hence, by Proposition 1.3, $G / Q$ is amenable, which proves the theorem.

Let $(X, \mathfrak{M}, m)$ be a measure space; that is, $\mathfrak{M}$ is a $\sigma$-algebra of subsets of $X$, and $m$ is a (countably additive) measure defined on $\mathfrak{M}$. Let $G$ be a group acting on $X$ preserving $\mathfrak{M}$ and $m$; that is, for all $g \in G$ and $E \in \mathfrak{M}, g E \in \mathfrak{M}$ and $m(g E)=m(E)$. The $G$-action is said to be weakly measurable if for any subgroup $H$ of $G, F_{H} \in \mathfrak{M}$. A weakly measurable action is said to be essentially factorable through a normal subgroup $H$ of $G$ if $m\left(X-F_{H}\right)=0$. A finitely additive measure $\mu$ on $(X, \mathfrak{P}(X))$ is said to extend the measure $m$ on $(X, \mathfrak{M})$ if $\mu(E)=m(E)$ for all $E \in \mathfrak{M}$.

THEOREM 1.4. Let $G$ be a group acting on a measure space $(X, \mathfrak{M}, m)$, preserving $\mathfrak{M}$ and $m$. Suppose $m(X)=1$ and the $G$-action is weakly measurable. Suppose also that $\left\{G_{x} \mid x \in X\right\}$ and $\left\{F_{H} \mid H\right.$ a subgroup of $\left.G\right\}$ are of type $\mathfrak{L}$. Then there exists a $G$-invariant finitely additive measure $\mu$ on $(X, \mathfrak{P}(X))$ extending the measure $m$ on $(X, \mathfrak{M})$ if and only if the $G$-action is essentially factorable through a normal subgroup $Q$ such that $G / Q$ is amenable.

ProOF. Suppose there exists a $G$-invariant finitely additive measure $\mu$ on $(X, \mathfrak{P}(X))$ extending $m$. By Theorem 1.1 there exists a normal subgroup $Q$ of $G$ such that $G / Q$ is amenable and $\mu\left(F_{Q}\right)=1$. Since $F_{Q} \in \mathfrak{M}$ and $\mu$ extends $m$, we get $m\left(F_{Q}\right)=1$, that is, the action is essentially factorable through $Q$.

Conversely, suppose the action is essentially factorable through a normal subgroup $Q$ such that $G / Q$ is amenable. By a well-known result (cf. $[3$, Theorem 5.1]) the measure $m_{Q}$ on $\left(F_{Q}, \mathfrak{M}_{Q}\right)$, where $\mathfrak{M}_{Q}=\left\{E \cap F_{Q} \mid E \in \mathfrak{M}\right\}$, defined by $m_{Q}(E)=m(E)$ for all $E \in \mathfrak{M}_{Q}$ extends to a $G / Q$-invariant (under the factor action) finitely additive measure $\mu_{Q}$ on $\left(F_{Q}, \mathfrak{P}\left(F_{Q}\right)\right)$. Put $\mu(E)=\mu_{Q}\left(E \cap F_{Q}\right)$, for all $E \in \mathfrak{P}(X)$. Then $\mu$ is a $G$-invariant finitely additive measure on $(X, \mathfrak{P}(X))$ extending the measure $m$ on $(X, \mathfrak{M})$.

2. Examples. We now apply Theorems 1.1 and 1.4 to various situations.

(i) Let $X=S^{n}$, the $n$-dimensional sphere (of unit vectors in $\mathbf{R}^{n+1}$, with respect to the usual norm) and let $G$ be a subgroup of $O(n+1)$, the orthogonal group, acting as isometries of $S^{n}$. For each $x \in S^{n}, G_{x}=G \cap O(n+1)_{x}$. Since evidently $\left\{O(n+1)_{x} \mid x \in S^{n}\right\}$ is evidently of type $\mathfrak{L}$, so is $\left\{G_{x} \mid x \in S^{n}\right\}$. On the other hand, for each subgroup $H \subset G \subset O(n+1), F_{H}$ is a compact differentiable submanifold 
with finitely many connected components. It follows therefore that $\left\{F_{H} \mid H\right.$ a subgroup of $G\}$ is of type $\mathfrak{L}$. Hence we get the following.

COROLLARY 2.1. (a) Let $\mu$ be a G-invariant finitely additive probability (or finite) measure on $\left(S^{n}, \mathfrak{P}\left(S^{n}\right)\right)$. Then there exists a normal subgroup $Q$ of $G$ such that $G / Q$ is amenable and $\mu\left(S^{n}-Y\right)=0$, where $Y=F_{Q}=\left\{x \in S^{n} \mid q x=x\right.$ for all $q \in Q\}$. $Y$ is a $G$-invariant set and $\{g / Y \mid g \in G\}$, the group of restrictions of elements of $G$ to $Y$, is an amenable group.

(b) There exists a $G$-invariant finitely additive measure $\mu$ on $\left(S^{n}, \mathfrak{P}\left(S^{n}\right)\right)$ extending the standard $(O(n+1)$-invariant $)$ measure $m$ on $\left(S^{n}, \mathfrak{B}\right)$, where $\mathfrak{B}$ is the $\sigma$-algebra of Borel sets, if and only if $G$ is amenable.

Proof. (a) Let $Q$ be as given by Theorem 1.1. Then since $Q$ is normal, $Y=F_{Q}$ is $G$-invariant. Since $Q$ fixes each $y \in Y$ the group $\{g / Y \mid g \in G\}$ is a quotient of $G / Q$ and therefore amenable (cf. [2]).

(b) If such an extension exists, then by Theorem 1.4 there exists a normal subgroup $Q$ such that $G / Q$ is amenable and $m\left(S^{n}-F_{Q}\right)=0$. The latter condition implies that $F_{Q}$ is dense in $S^{n}$ and, since it is closed, we have $F_{Q}=S^{n}$. But identity is the only isometry fixing all points. Hence $Q$ is trivial and consequently $G$ is amenable. Conversely, if $G$ is amenable, then by a well-known result (cf. Theorem 5.1 of $[3])$ there exists a $G$-invariant finitely additive measure on $\left(S^{n}, \mathfrak{P}\left(S^{n}\right)\right)$ extending $m$.

Part (b) was proved earlier by Wagon (cf. [6]). He also noted part (a) in the particular case $n=2$ and conjectured its validity for all $n$ (cf. [6]); the corollary establishes the conjecture. (We should, however, note the following: In the statement of the conjecture in [6, p. 81], it is not explicit whether the measure is meant to be finite. But in the particular case of $S^{2}$ which is settled in [6] and from which the author motivates the conjecture, the measure is implicitly assumed to be finite (cf. Theorems 6 and 7 and Proposition 1 in [6]). Whether the conjecture is true for $\sigma$-finite additive measures is not clear, even for $S^{2}$.)

In [6] it is actually shown (cf. Theorem 7 of [6]) that for any nonamenable group $G$ of isometries of $S^{2}$ there does not exist any (finite) $G$-invariant finitely additive measure on $\left(S^{2}, \mathfrak{P}\left(S^{2}\right)\right)$. We note that if $G$ is a nonamenable subgroup of $O(3)$ then the natural action of $G$ on $\mathbf{R}^{3}$ is irreducible (that is, there is no proper nontrivial invariant subspace). The above-mentioned assertion from [6] generalizes to the following.

COROLlaRY 2.2. Let $G$ be a nonamenable subgroup of $O(n+1)$ acting irreducibly on $\mathbf{R}^{n+1}$. Then there does not exist any $G$-invariant finitely additive probability measure on $\left(S^{n}, \mathfrak{P}\left(S^{n}\right)\right)$.

ProOF. If possible let $\mu$ be such a finitely additive measure. By Corollary 2.1 there exists a normal subgroup $Q$ of $G$ such that $G / Q$ is amenable and $\mu\left(F_{Q}\right)=1$. Evidently, $F_{Q}=S^{n} \cap W$, where $W=\left\{x \in \mathbf{R}^{n+1} \mid q x=x\right.$ for all $\left.q \in Q\right\}$. Since $Q$ is normal $W$ is a $G$-invariant subspace of $\mathbf{R}^{n+1}$. By irreducibility either $W=(0)$ or $\mathbf{R}^{n+1}$. The former is impossible since $F_{Q}$ would then be empty, while actually $\mu\left(F_{Q}\right)>0$. On the other hand, if $W=\mathbf{R}^{n+1}$ then $F_{Q}=S^{n}$, which means $Q$ must be the trivial subgroup; but in that case $G$ must be amenable-a contradiction to the hypothesis. Hence there cannot exist such a finitely additive invariant measure. 
(ii) The above examples generalize in a natural way to homogeneous spaces of compact Lie groups. Let $\mathfrak{G}$ be a compact Lie group and $\mathfrak{S}$ be a closed subgroup of $\mathfrak{G}$. Let $X=\mathfrak{G} / \mathfrak{S}$ and let $G$ be a subgroup of $\mathfrak{G}$ acting on $X$ on the left. Then arguments as above yield the following.

COROLLARY 2.3. (a) Let $\mu$ be a G-invariant finitely additive probability measure on $(X, \mathfrak{P}(X))$. Then there exists a normal subgroup $Q$ of $G$ such that $G / Q$ is amenable and $\mu\left(X-F_{Q}\right)=0$.

(b) There exists a $G$-invariant finitely additive measure $\mu$ on $(X, \mathfrak{P}(X))$ extending the $\mathfrak{G}$-invariant (countably additive) probability measure on $(X, \mathfrak{B})$, where $\mathfrak{B}$ is the $\sigma$-algebra of Borel sets, if and only if $G$ is amenable.

(iii) Similar arguments may be made for homogeneous spaces of algebraic groups: Let $\mathfrak{G}$ be an algebraic $\mathbf{R}$-group (the group of $\mathbf{R}$-elements of an algebraic group defined over $\mathbf{R}$ ), e.g. $\mathrm{GL}(n, \mathbf{R})$. Let $\mathfrak{S}$ be an algebraic $\mathbf{R}$-subgroup of $\mathfrak{G}$. Let $X=\mathfrak{G} / \mathfrak{S}$ and let $G$ be a subgroup of $\mathfrak{G}$ acting on $X$ on the left. Then for each $x \in X, G_{x}=G \cap \mathfrak{G}_{x} .\left\{\mathfrak{G}_{x} \mid x \in X\right\}$ is a family of algebraic $\mathbf{R}$-subgroups and therefore it is of type $\mathfrak{L}$. Hence so is $\left\{G_{x} \mid x \in X\right\}$. On the other hand, for any subgroup $H, F_{H}$ is the set of $\mathbf{R}$-elements of an algebraic variety. Therefore $\left\{F_{H} \mid H\right.$ a subgroup of $G\}$ is of type $\mathfrak{L}$. It follows that Corollary 2.2(a) holds verbatim for $G$ and $X$ as above. In general, $(X, \mathfrak{B})$, where $\mathfrak{B}$ is the Borel $\sigma$-algebra, may not carry any finite $G$-invariant (countably additive) measure. If it does then Corollary 2.2(b) also holds verbatim for those $G$ and $X$. We note that since compact Lie groups are algebraic $\mathbf{R}$-groups these results generalise Corollary 2.3. Similarly the following generalises Corollary 2.1 .

COROllaRY 2.4. Let $G$ be a subgroup of $\operatorname{GL}(n, \mathbf{R})$. Let $X$ be either $\mathbf{R}^{n}-(0)$ or $S^{n-1}$ or $\mathbf{P}^{n-1}((n-1)$-dimensional projective space $)$ and consider the natural $G$-action on $X$. Let $\mu$ be a $G$-invariant finitely additive probability measure on $(X, \mathfrak{P}(X))$. Then there exists a normal subgroup $Q$ of $G$ such that $G / Q$ is amenable and $\mu\left(F_{Q}\right)=1$. In particular, if $G$ is nonamenable, and the action of $G$ on $\mathbf{R}^{n}$ is irreducible (e.g. if $G=\mathrm{SL}(n, \mathbf{Z}))$ then there does not exist any $G$-invariant finitely additive probability (or finite) measure on $(X, \mathfrak{P}(X))$.

In the case of $X=\mathbf{R}^{n}-(0)$ or $\mathbf{P}^{n-1}$ the isotropy subgroups for the (transitive) $\operatorname{GL}(n, \mathbf{R})$-action are algebraic $\mathbf{R}$-subgroups and the result follows from the above remarks. For $S^{n-1}$ it may be deduced either from the result for $\mathbf{P}^{n-1}$ or directly from Theorem 1.1. If the action of $G$ on $\mathbf{R}^{n}$ is irreducible, then as in the proof of Corollary 2.2 we see that for any normal subgroup $H$ of $G, F_{H}$ must be either empty or $X$ (for any $X$ as above). Hence for such a $G$ there cannot exist any invariant finitely additive measure on $(X, \mathfrak{P}(X))$ unless it is amenable.

In $[\mathbf{1}]$ we proved the analogous assertion for the actions of subgroups of $\operatorname{GL}(n, \mathbf{Z})$ acting on $\mathbf{T}^{n}$, the $n$-dimensional torus, as the group of automorphisms. In that case, however, Theorems 1.1 and 1.4 do not apply since $G_{x}$ for $x \in \mathbf{T}^{n}$ cannot in general be expressed as $G \cap \mathfrak{G}_{x}$ for some algebraic group $\mathfrak{G}$; in fact $\left\{G_{x} \mid x \in \mathbf{T}^{n}\right\}$ is not of type $\mathfrak{L}$. The proof there uses, together with the ideas underlying the proof of Theorem 1.1, certain specific features of the torus. 


\section{REFERENCES}

1. S. G. Dani, On invariant finitely additive measures for automorphism groups of tori, Trans. Amer. Math. Soc. (to appear).

2. F. P. Greenleaf, Invariant means on topological groups, Van Nostrand Reinhold, New York, 1969.

3. J. Mycielski, Finitely additive invariant measures. I, Colloq. Math. 42 (1979), 309-318.

4. J. Rosenblatt, Uniqueness of invariant means for measure-preserving transformations, Trans. Amer. Math. Soc. 265 (1981), 623-636.

5. A. Tarski, Algebraische Fassung des Massproblems, Fund. Math. 31 (1938), 47-66.

6. S. Wagon, Invariance properties of finitely additive measures on $\mathbf{R}^{n}$, Illinois J. Math. 25 (1981), 74-86.

7.

School of Mathematics, Institute for Advanced Study, Princeton, New JERSEY 08540

Current address: School of Mathematics, Tata Institute of Fundamental Research, Homi Bhabha Road, Bombay 400005, India 